\title{
NONINVASIVE METHODS IN EVALUATION OF PORTAL HYPERTENSION
}

\author{
Edlira Elezaj $^{1 *}$, Adriana Babameto ${ }^{2}$ \\ ${ }^{1 *}$ Polyclincic of Specialities No.2 Tirana, Tirana, Albania; \\ ${ }^{2}$ University Hospital "Mother Teresa", Department of Gastrohepatology at Tirana, Albania; \\ *Corresponding author Edlira Elezaj, email: edliraelezaj@gmail.com; \\ Received May, 2018; Accepted May, 2018; Published June, 2018; \\ doi: https://doi.org/10.31407/ijees8313 \\ UOI license: http://u-o-i.org/1.01/ijees/35941258
}

\begin{abstract}
Non-invasive methods that can be used reliably to determine the presence and estimate the degree of portal hypertension have been in great demand for at least 30 years. Despite substantial efforts to generate such a method, up until a few years ago only disappointing and unsatisfactory results were obtained. Since the primary cause of portal hypertension is the mechanical increase in intrahepatic resistance due to fibrosis and distortion of liver architecture, it is reasonable to assume that non-invasive parameters of liver fibrosis may indicate the presence of portal hypertension. PHT is a robust outcome measure which has proven prognostic significance in chronic liver disease and the potential for use in monitoring disease progression and treatment efficacy. In this paper we have outlined the pathogenesis of PHT and discussed a range of candidate serum biomarkers that have been identified. At present, transient elastography appears to represent the most promising noninvasive technique that could potentially replace HVPG measurement for PHT or endoscopy for variceal detection. The potential role of serum markers for the evaluation of PHT it is increasingly being assessed in prospective clinical studies. Further advances in our understanding of the underlying mechanisms responsible for the development and progression of PHT will continue to reveal additional biomarker targets.
\end{abstract}

Key words: portal hypertension, non invasive methods, elastography, serum biomarkers 